\title{
Distribución y variabilidad del registro arqueológico de superficie en Cerro Dragón (provincia de Chubut) y Koluel Kaike-Piedra Clavada (provincia de Santa Cruz)
}

\author{
Mariano Carlos Del Papa* \\ Dario Hermo**
}

\begin{abstract}
DEL PAPA, M.C.; HERMO, D. Distribución y variabilidad del registro arqueológico de superficie en Cerro Dragón (provincia de Chubut) y Koluel Kaike-Piedra Clavada (provincia de Santa Cruz). R. Museu Arq. Etn., 28: 70-85, 2017.
\end{abstract}

Resumen: Los estudios que se encuentran enmarcados dentro de la denominada arqueología de contrato, específicamente los referidos a estudios de impacto ambiental, han revisto en los últimos años una fuente importante de información sobre las características del patrimonio arqueológico de diferentes regiones de la República Argentina. En este contexto, los resultados presentados en este trabajo pertenecen al Estudio de Línea de Base correspondiente a dos áreas de explotación petrolera. Dichas áreas se hallan localizadas en la denominada Cuenca Hidrocarburífera del golfo San Jorge, (Cerro Dragón, provincia de Chubut, y Koluel Kaike-Piedra Clavada, Provincia de Santa Cruz). La información obtenida, como producto de la prospección superficial permitió realizar un conjunto de análisis que permitió la caracterización del registro arqueológico de una importante área sobre la que prácticamente no existen antecedentes. Se analizó la distribución de diferentes tipos de hallazgos, las materias primas utilizadas y aspectos de las tecnologías representadas en la muestra. Se calculó la riqueza y homogeneidad y la posible asociación entre diferentes unidades de paisaje y las diferentes categorías analíticas utilizadas. Los resultados obtenidos muestran la existencia de una distribución del registro arqueológico en superficie que se expresa de forma heterogénea entre las diferentes unidades del paisaje analizadas, encontrándose las mayores densidades en aquellas áreas cercanas a recursos críticos tales como fuentes de agua.

Palabras clave: Arqueología de contrato; Patagonia; Golfo San Jorge; Cerro Dragón; Koluel Kaike-Piedra Clavada.

\footnotetext{
* División Antropología, Facultad de Ciencias Naturales y Museo, Universidad de La Plata (UNLP).

<mdelpapa@fcnym.unlp.edu.ar>

** Consejo Nacional de Investigaciones Científicas y

Técnicas (Coniciet). División Arqueología, Facultad de

Ciencias Naturales y Museo, Universidad de La Plata

(UNLP).<dhermo@fcnym.unlp.edu.ar>
}

\section{Introducción}

E $n$ el presente trabajo se exponen

Clos resultados obtenidos a partir

de las tareas de relevamiento arqueológico realizados en las áreas Cerro Dragón (provincia de Chubut) y Koluel Kaike-Piedra Clavada 
(provincia de Santa Cruz), correspondiente a la porción oriental de la cuenca petrolifera del Golfo San Jorge. A menudo, este tipo de trabajos por contrato suele quedar documentado en informes que circulan entre empresas, consultoras y entes gubernamentales de cultura, patrimonio y/o turismo. Sólo en algunas oportunidades la información generada mediante tal estrategia de trabajo arqueológico suele ser publicada en forma de información de base que permita a otros colegas conocer, comparar y valorar algunas de las características del registro arqueológico. La publicación de los casos tenidos en cuenta en este trabajo, es decir áreas que cuentan con investigaciones sistemáticas puntuales o muy recientes, puede representar un avance en el conocimiento de algunas particularidades de la utilización del espacio patagónico y sus recursos por parte de poblaciones prehispánicas.

El área correspondiente a Cerro Dragón (en adelante $\mathrm{CD}$ ) se encuentra localizada en el SE de la provincia de Chubut (Fig. 1). El predio abarca un polígono de forma irregular, con una superficie total de $3.480 \mathrm{~km}^{2}$ y cuyos límites son arbitrarios, con excepción del límite norte representado por el margen izquierdo del río Chico y el lago Colhué Huapi. El paisaje está dominado por geoformas caracterizadas por una homogeneidad en altitud, y que en conjunto constituyen un sistema de drenaje que aporta sus afluentes al río Chico y al lago Colhué Huapi.

El área denominada Koluel Kaike-Piedra Clavada (en adelante KK-PC) se encuentra localizada en el NE de la provincia de Santa Cruz (Fig. 1). El predio ocupa una superficie total de $289 \mathrm{~km}^{2}$ y también en este caso el perímetro tiene una forma arbitraria con excepción del límite sur representado por el margen norte del río Deseado. La geomorfología está dominada por la meseta, donde se registran las mayores altitudes. Desde allí descienden numerosos cañadones formados por cauces temporarios y mallines que depositan su caudal en el río Deseado. Por último, la terraza del río Deseado corresponde al sector que se halla más bajo, prácticamente al nivel del lecho, por este motivo, además de recibir el caudal de la meseta y la cuenca, su sector inmediatamente lindante al río está dominado los regímenes hidrológicos del mismo, convirtiéndolo en una llanura de inundación.

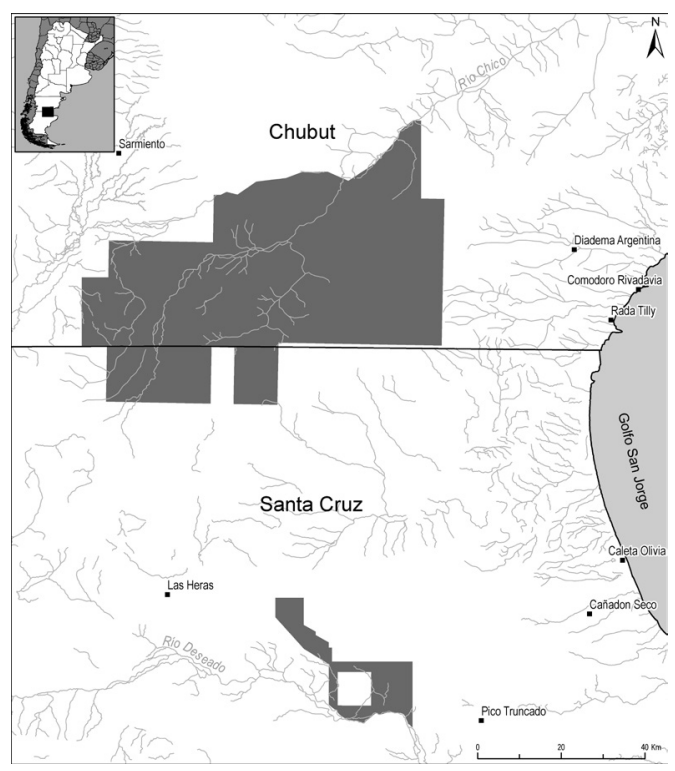

Fig 1. Localización de las áreas de estudio.

Fuente: Cartografía elaborada a partir del SIG 250 del Instituto Geográfico Nacional (IGN)

El objetivo general que orientó los trabajos de campo fue caracterizar de manera general del patrimonio cultural arqueológico observable en superficie, que permita una base de información local que pueda ser usada en futuros estudios (i.e. ambientales, arqueológicos, etc.). El objetivo particular del presente trabajo es identificar patrones de distribución y variabilidad del registro arqueológico de superficie y reconocer procesos que pudieran dar cuenta de dichos patrones.

\section{Antecedentes}

Uno de los principales intereses del presente trabajo es el de exponer resultados correspondientes a áreas que, en términos generales, no cuentan con información 
Distribución y variabilidad del registro arqueológico de superficie en Cerro Dragón (provincia de Chubut) y Koluel Kaike-Piedra Clavada (provincia de Santa Cruz)

R. Museu Arq. Etn., 28: 70-85, 2017.

arqueológica previa registrada de manera sistemática. Para el área correspondiente al sudeste de la provincia de Chubut, los principales antecedentes se centran en las investigaciones realizadas en los lagos Musters y Colhué Huapi (Outes 1905; Vignati 1950; Menghin 1952; Moreno \& Pérez Ruiz 2010) que fueron orientadas a generar conocimiento sobre las ocupaciones prehistóricas del área. En los últimos años se han realizado importantes esfuerzos para generar, desde diferentes enfoques, los primeros esquemas cronológicos, tanto para el lago Musters (Zubimendi \& Moreno 2014), el lago Colhué Huapi (García Guraieb et al. 2009) y el río Chico (Pérez de Micou 2009).

Las recientes investigaciones llevadas a cabo en el lago Colhué Huapi evidencian ocupaciones de cazadores-recolectores que indican un énfasis en la pesca dulceacuícola (i.e. perca, pejerrey patagónico) (Moreno, Pérez
Ruiz \& Ramírez Rozzi 2016). Entre la cultura material asociada a los sitios registrados en la planicie de inundación en el noroeste y sureste del lago Colhué Huapi (Moreno, Pérez Ruiz \& Ramírez Rozzi 2016) cabe destacar la presencia de puntas de proyectil y raspadores (en muchos casos microlíticos Fig. 2d), fragmentos de cerámica y de placas grabadas. Resulta llamativa la presencia de pesos de red (Reyes \& Svoboda 2016), distinguibles como "rodados aplanados con muescas en polos opuestos, dispuestos tanto de forma aislada como en concentraciones de hasta 15 artefactos" (Moreno, Pérez Ruiz \& Ramírez Rozzi 2016). Este tipo de objetos ha sido relevado asimismo en colecciones arqueológicas provenientes de los lagos Musters y Colhué Huapi (Moreno \& Pérez Ruiz, 2010). Además, se han registrado estructuras de piedra, asignadas a parapetos y chenques, y petroglifos sobre bloques de piedra (Moreno, Pérez Ruiz \& Ramírez Rozzi 2016).

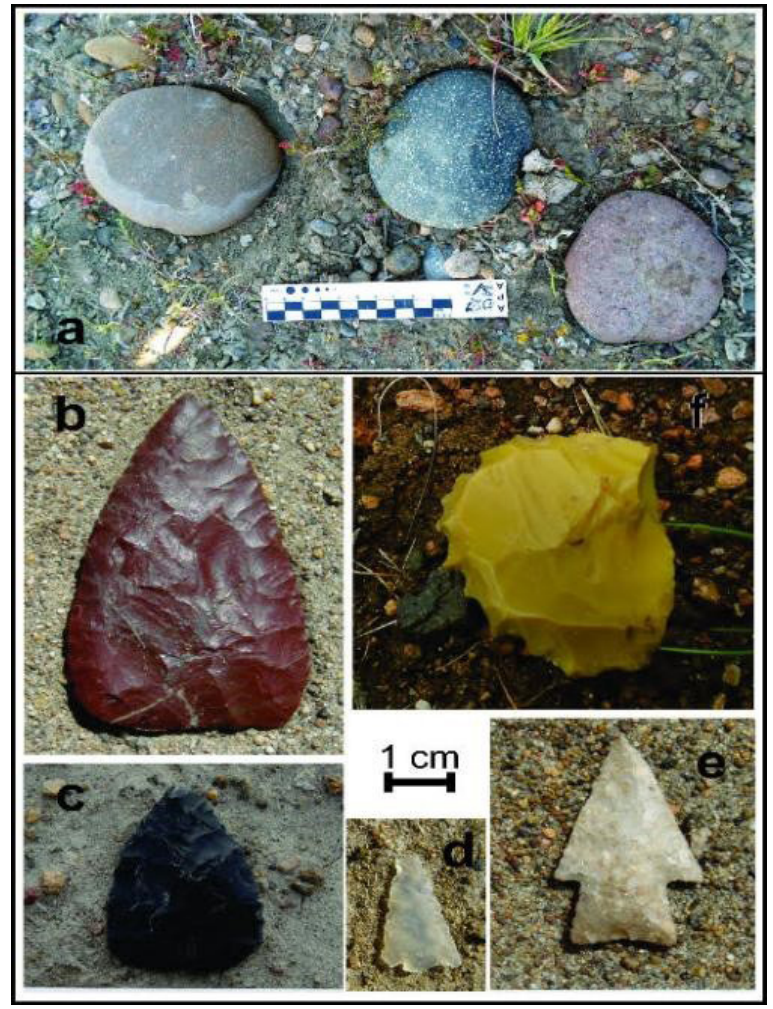

Fig.2. Instrumental lítico registrado en superficie durante tareas de campo.

Fuente: Las imágenes fueron originadas durante las tareas de campo. 
En este contexto de investigaciones arqueológicas, el área del yacimiento Cerro Dragón representa una porción significativa de superficie, posee una gran variedad de microambientes con presencia de recursos críticos tales como el agua (principalmente representada por una compleja red de cauces, tributarios al río Chico y al lago Colhué Huapi), áreas de abrigo (como se observa en los cañadones) y disponibilidad de rocas (i.e. madera petrificada, rocas volcánicas) utilizadas como materia prima para la manufactura artefactual. No obstante, hasta la actualidad no se han realizado investigaciones arqueológicas sistemáticas (con excepción de las sintetizadas en el párrafo anterior) que permitan evaluar en qué medida las poblaciones del pasado utilizaron dichos recursos.

En lo que respecta al sector Koluel Kaike-Piedra Clavada, los principales antecedentes provienen de investigaciones realizadas en sectores relacionados con la cuenca del río Deseado. Cabe destacar que al sur de la cuenca media del río Deseado se extiende el Macizo del Deseado. Las investigaciones en esta área han mostrado que desde las primeras ocupaciones humanas que se iniciaron hace aproximadamente 13.000 años antes del presente. Este sector ha sido ocupado recurrentemente hasta el Holoceno tardío. Los sitios arqueológicos de Los Toldos (A. Cardich, L. Cardich \& Hajduk 1973), Piedra Museo (Miotti 1996; Miotti \& Salemme 2003) y Cueva Maripe (Miotti et al. 2014) muestran diferentes sucesiones de ocupaciones humanas que abarcan todo el Holoceno. En las nacientes del río Deseado, se ubica el área Río Pinturas, otra de las áreas más prolíficas en tradición de investigaciones que han establecido un esquema ocupacional desde momentos tempranos ca. 9.300 años antes del presente (Gradin 1973; Gradin, Aschero \& Aguerre, 1987; Gradin \& Aguerre 1994).

En similitud con los estudios que presentamos aquí, caben destacar los trabajos de línea de base de un estudio de impacto ambiental y social realizados por Norma Ratto y Dolores Carniglia en el sector santacruceño al norte del río Deseado (Ratto \& Carniglia 2013). El área analizada se extendía desde la costa del golfo San Jorge hasta el oeste de la ciudad de Las Heras, un espacio para el que no contaron con información antecedente de investigaciones arqueológicas sistemáticas, con excepción de las realizadas en la costa (Ratto \& Carniglia 2013). Los análisis distribucionales y taxonómicos realizados les permiten concluir que "a pesar de los intensos procesos de formación de origen antrópico y natural, fue posible definir una estructura del registro caracterizada por su baja abundancia, distribución azarosa y diversidad baja a media, independientemente de la geoforma relevada en los distintos sectores definidos" (Ratto \& Carniglia 2013: 492).

\section{Metodología}

Para el reconocimiento de las propiedades distribucionales del registro arqueológico de las áreas en estudio se llevó a cabo un diseño de prospección fundamentado en una metodología distribucional (Borrero, Lanata \& Ventura 1992; Ebert 1992; Belardi 2005). En la puesta a punto de este diseño se consideró dividir a cada área en diferentes subáreas, utilizando como criterio las unidades del paisaje que las constituyen. A cada una de estas subáreas se les asignó una cantidad proporcional de transecta de muestreo. Este tipo de estrategia nos permitió generar un cuadro distribucional de los restos arqueológicos registrados para comparar la densidad de cada subárea respecto de las demás. De esta manera, la variabilidad observada en la densidad puede reflejar, de forma relativa, el carácter y la frecuencia de la utilización del espacio (Foley 1981; Dunnell \& Dancey 1983). A la vez, la utilización de las unidades de paisaje posibilitó el análisis de la variabilidad en la representación de frecuencias de hallazgos de restos arqueológicos según diferencias en el ambiente.

El concepto de unidad del paisaje se define como un sector discreto y perceptible del espacio que presenta características geográficas y geomorfológicas específicas y homogéneas 
Distribución y variabilidad del registro arqueológico de superficie en Cerro Dragón (provincia de Chubut) y Koluel Kaike-Piedra Clavada (provincia de Santa Cruz)

R. Museu Arq. Etn., 28: 70-85, 2017.

(Borrero, Lanata \& Ventura 1992). Para este trabajo las unidades del paisaje se definieron teniendo en cuenta dos aspectos, la topografía y los sistemas de cauces permanentes y efímeros, como también otros cuerpos de agua. De esta manera, el área correspondiente a CD fue

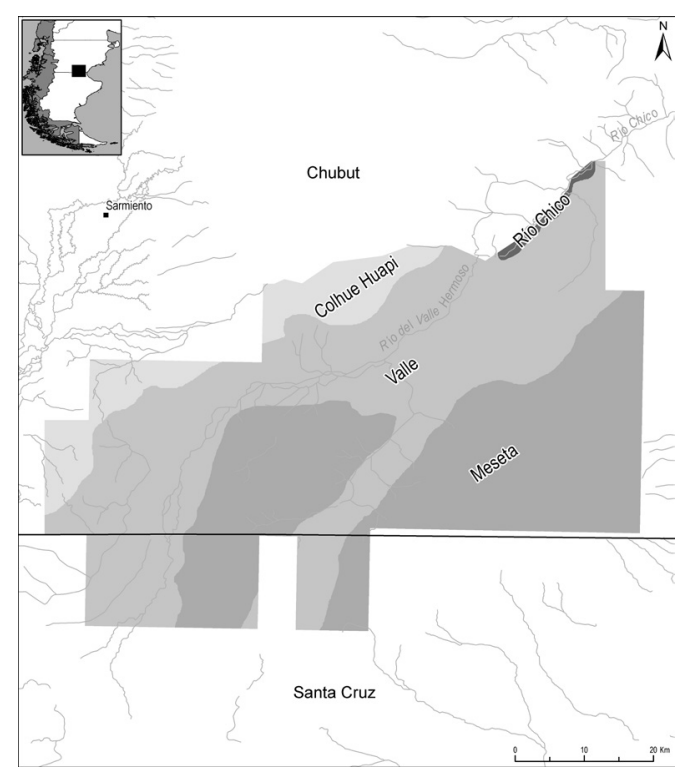

Fig. 3. Área Cerro Dragón con sus correspondientes unidades de paisaje.

Fuente: Cartografía elaborada a partir del SIG 250 del Instituto Geográfico Nacional (IGN)

El objetivo de las prospecciones arqueológicas en campo es el de aplicar un conjunto de técnicas para optimizar las probabilidades de detección de materiales culturales, que conforman el registro arqueológico/histórico, en un espacio determinado (Gallardo \& Cornejo 1986). En este sentido, el trabajo de campo consistió en el relevamiento superficial de las áreas conformadas por las áreas de KK-PC (prov. de Santa Cruz) y CD (prov. de Chubut). Para las tareas de relevamiento se siguieron métodos sistemáticos que fueron planteados por diferentes autores (Gallardo \& Cornejo 1986, Borrero, Lanata \& Ventura 1992, Scheinsohn 2001, Herrera, Cuenca \& Pérez 2009, entre otros). Estos métodos de cuantificación dividido en cuatro unidades de paisaje: meseta, valle, lecho seco (sur del lago Colhué Huapi) y río Chico (Fig. 3); mientras que KK-PC fue dividida en tres unidades de paisaje, las mismas son meseta, terraza y valle del río Deseado (Fig. 4).

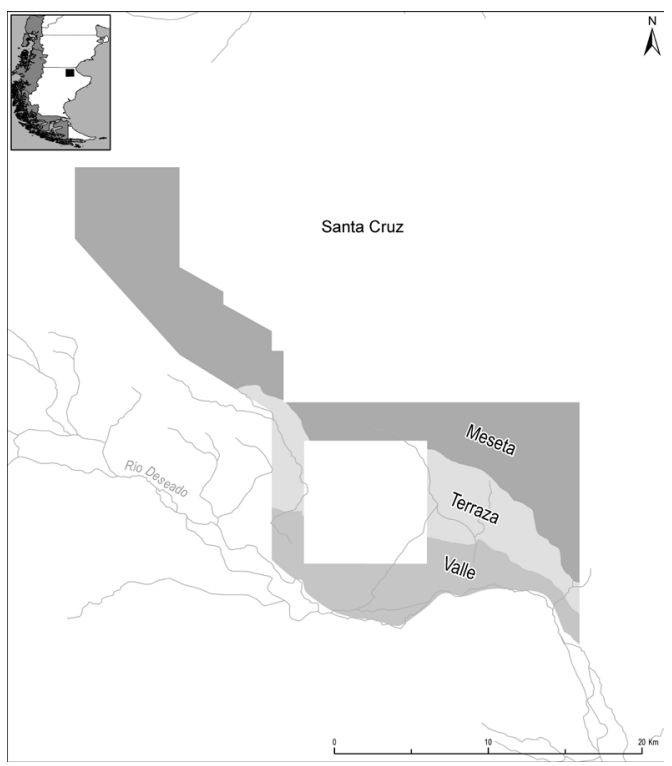

Fig. 4. Área Koluel Kaike-Piedra Clavada con sus correspondientes unidades de paisaje.

Fuente: Cartografía elaborada a partir del SIG 250 del Instituto Geográfico Nacional (IGN)

permiten la mayor cobertura posible de grandes territorios y de una manera estadísticamente válida, por medio de la aplicación de prospección por transectas sistemáticas (Judge, Ebert \& Hitchcock 1975; S. Plog, F. Plog \& Wait 1978).

Las transectas fueron diseñadas para un muestreo de tipo estratificado, en el que el área a relevar es dividida en un cierto número de subáreas o estratos, en orden de homogeneizar la muestra. Más tarde, cada una de ellas fue muestreada independientemente, según la proporción del área total que representen. Para este trabajo se definieron estratos en base a la topografía del área de estudio, es decir que cada estrato corresponde a una de las unidades de paisaje mencionadas 
anteriormente. Se implementaron dos tipos de transectas: intensivas (probabilísticas) y prospecciones extensivas (no probabilísticas) (Borrero, Lanata \& Ventura 1992; Foley 1981). Las prospecciones intensivas (probabilísticas) permiten obtener una visión más detallada de la distribución de ítems arqueológicos y su relevancia para el área de estudio. Bajo esta metodología se realizó un total de 85 transectas de $5000 \mathrm{~m}$ de longitud, para el yacimiento CD y 27 transectas de $3000 \mathrm{~m}$ de longitud, para el yacimiento KK-PC; y de $30 \mathrm{~m}$ de ancho para ambos casos, respetando las proporciones de representatividad para cada estrato. Por otro lado, las prospecciones extensivas (no probabilísticas) se utilizaron para relevar todos aquellos sectores, que por antecedentes bibliográficos y por la información obtenida mediante las prospecciones intensivas, resultan de particular interés distribucional. En este sentido, se realizó un total de 19 transectas de $5000 \mathrm{~m}$ de longitud para el yacimiento CD y 10 transectas de $3000 \mathrm{~m}$ de longitud para el yacimiento KK-PC; y de $30 \mathrm{~m}$ de ancho, respetando las proporciones de representatividad para cada estrato. La distribución del registro arqueológico de superficie fue documentada según su disposición en el terreno, teniendo en cuenta las categorías analíticas de sitio, concentración y hallazgo aislado (Borrero, Lanata \& Ventura 1992).

Una vez obtenidos los datos se realizó el cálculo de densidad artefactual y el cálculo de los índices de riqueza y homogeneidad. Para conocer si existe algún tipo de dependencia entre atributos como categorías analíticas, tecnología y materia prima en relación a las unidades de paisaje descriptas, se llevó a cabo un análisis de $\mathrm{Chi}^{2}$ con tablas de contingencia. La comparación de la variabilidad de los conjuntos se calculó mediante el uso del índice de riqueza y de homogeneidad (Shannon \& Weaver 1949; Zar 1974; Pielou 1977). Estos índices muestran la diversidad de los conjuntos minimizando la incidencia que el tamaño de las muestras tiene sobre las comparaciones entre ellas (Guráieb 1999; Bozzuto 2013). Los cálculos fueron realizados en base al número mínimo de desechos (Aschero, Manzi \& Gómez 1994), teniendo en cuenta la suma de los artefactos enteros y los fragmentados con talón, ya que de esta manera se evita la sobrestimación de determinadas categorías en la muestra analizada.

\section{Resultados}

\section{Caracterización del registro arqueológico}

Durante los trabajos de campo en Cerro Dragón se relevaron un total de 66 transectas intensivas (probabilísticas) correspondiente a una superficie de $10,35 \mathrm{~km}^{2}$ y 19 transectas extensivas (no probabilísticas) que cubrieron una superficie de 2,85 $\mathrm{km}^{2}$ (total 13,2 $\mathrm{km}^{2}$ ). Por otro lado, el área de KK-PC se realizaron un total de 17 transectas intensivas y 10 transectas extensivas que cubrieron una superficie de $1,53 \mathrm{~km}^{2}$ y 0,9 $\mathrm{km}^{2}$ (total 2,43 $\left.\mathrm{km}^{2}\right)$. Se registraron un total de $9150(\mathrm{n}=7974$ en CD y $n=1176$ en KK-PC) artefactos líticos, entre los que la clase más representada es la de los artefactos no formatizados ( $\mathrm{n}=8296$; de los cuales 7253 corresponden a CD y 1043 a KK-PC), seguida por los núcleos ( $\mathrm{n}=454$, 398 de CD y 56 de KK-PC) y los artefactos formatizados ( $n=400,323$ de CD y 77 de KK-PC). Estas frecuencias se distribuyen de diferente manera en las unidades de paisajes en las que se segmentó el espacio. En este sentido, las unidades que mayores frecuencias artefactuales presentaron son las de Valle en CD $(n=4407)$ y la de Cuenca en KK-PC $(\mathrm{n}=688)$ (véase Tabla 1 y Gráficos 1 y 2).

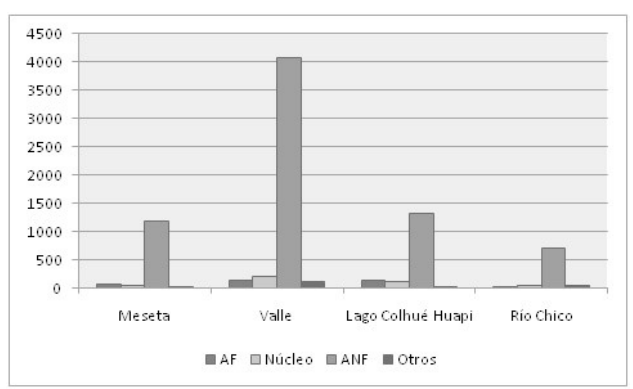

Gráfico 1. Tecnología CD. 
Distribución y variabilidad del registro arqueológico de superficie en Cerro Dragón (provincia de Chubut) y Koluel Kaike-Piedra Clavada (provincia de Santa Cruz)

R. Museu Arq. Etn., 28: 70-85, 2017.

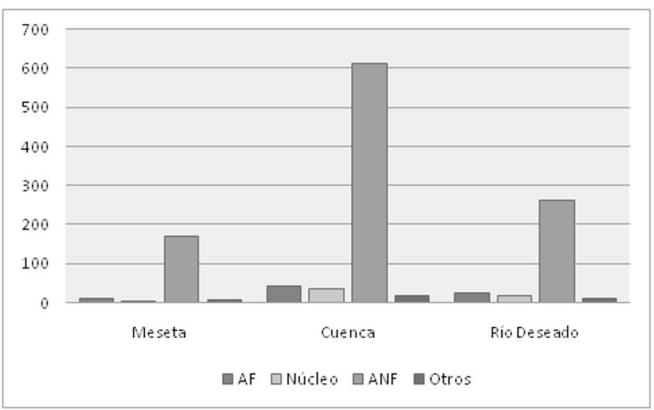

Gráfico 2. Tecnología KK.

Cabe destacar que para una mejor caracterización de la distribución de los AF se procedió a discriminar entre grupos tipológicos, aunque dado el tipo de registro en campo y las cantidades de material relevado, sólo se utilizaron los grupos tipológicos más representados (raspadores, raederas, puntas de proyectil y bolas), los demás artefactos formatizados fueron agrupados en la categoría Otros AF, que fue excluida de los cálculos de riqueza y homogeneidad (véase más adelante). Cabe destacar que durante las prospecciones en CD se detectaron pesas (Fig. 2a), que constituyen una evidencia particular por su infrecuente registro a nivel regional y que fueron incluidas en la categoría Otros AF. En los gráficos 1 e 2 se observa que las frecuencias artefactuales se encuentran, como es esperable, se encuentra dominada por la presencia de ANF. La distribución de los hallazgos según la clase tipológica es diferente en cada unidad de paisaje. La unidad Meseta, tanto en CD como en KK-PC, fue la que contó con mayor cantidad de $\mathrm{km}^{2}$ prospectados, aunque con densidades artefactuales bajas. Las frecuencias de AF y Núcleos son relativamente mayores que en las áreas de densidad alta. Sin embargo, la proporción de AF y Núcleos es mayor aun en unidades que no son necesariamente las más densas Valle en CD y Cuenca en KK-PC, es decir en aquellos rasgos geomorfológicos intermedios.

En relación a la forma en que se distribuye el registro arqueológico, en un total de 218 loci se identificaron un total de 50 sitios, 83 concentraciones y 85 hallazgos aislados. Al considerar las frecuencias de estas categorías por áreas, en CD se relevaron 35 sitios, 58 concentraciones y 31 hallazgos aislados (Tabla 1 y Gráfico 3); mientras que para KK-PC estas frecuencias son de 15,25 y 24 respectivamente (Tabla 1 y Gráfico 4).

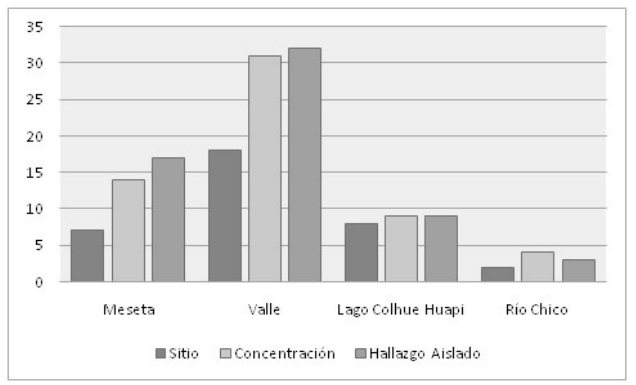

Gráfico 3. Tipo de hallazgos CD.

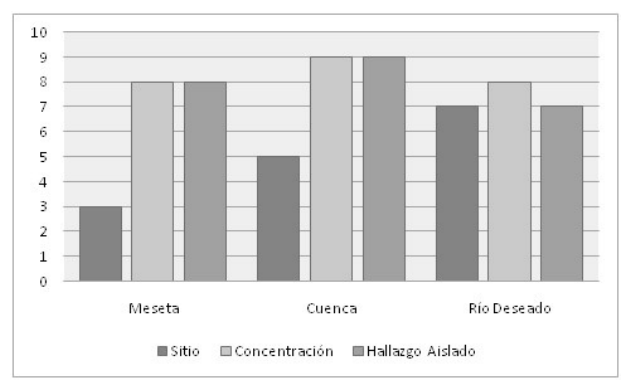

Gráfico 4. Tipo de hallazgos KK-PC.

Los gráficos 3 y 4 muestran las frecuencias de las categorías de agrupamiento del registro arqueológico registradas en las diferentes unidades de paisaje de cada una de las áreas analizadas. Se pueden destacar algunas particularidades, tales como que en CD, los hallazgos aislados son la forma de presentación del registro más numerosa en las unidades de paisaje Meseta y Valle, ya que en Lago Colhué Huapi se detectó la misma cantidad de concentraciones que de hallazgos aislados $(n=9)$, mientras que en Río Chico la categoría de agrupación del registro arqueológico fue la de concentración. Estas dos últimas situaciones son las que se registraron en KK-PC: en Meseta y Cuenca 
las concentraciones y los hallazgos aislados fueron registrados en la misma frecuencia ( $\mathrm{n}=8$ y 9 , respectivamente), mientras que en Río Deseado las concentraciones fueron el tipo de agrupación más frecuente. La categoría sitio estuvo presente en todas las unidades de paisaje en frecuencias menores a las alcanzadas por las demás categorías, aunque en áreas asociadas a los recursos hídricos (como Lago Colhué Huapi y Río Chico en CD, y Cuenca y Río Deseado en KK-PC), la diferencia entre la cantidad de sitios y de concentraciones y/o hallazgos aislados es menor que en las unidades de paisaje más alejadas al agua (Meseta y Valle en CD y Meseta en KK-PC).

Otro de los aspectos a abordar de los conjuntos tecnológicos es el uso de las materias primas. Durante los trabajos de campo se reconocieron ocho tipos de rocas, cuya distribución en las diferentes unidades de paisaje fue diferencial. De la categoría sílice, que incluye rocas micro a criptocristalinas opacas con alta silicificación, diferenciamos otra que denominamos "sílice negro" debido a su recurrente aparición en los conjuntos. En CD las materias primas más frecuentemente registrada fueron el basalto y las diferentes variedades de sílice (gráfico 5). Mientras que en las unidades Meseta y Valle el basalto es más frecuente que el sílice, en Lago Colhué Huapi y Río Chico el orden se invierte. La calcedonia es el tercer tipo de roca más representado en todas las unidades de paisaje, con excepción del antiguo lecho del lago. En esta unidad la jerarquía de uso de rocas se ubica en tercer lugar al sílice negro, seguido por la calcedonia. Las demás materias primas (xilópalo, ópalo y obsidiana) se encuentran en proporciones menores. La roca más registrada en cada una de las unidades de paisaje, el basalto adquiere relevancia por la altísima frecuencia en la que fue registrado en la unidad Valle. Por el contrario, en KK-PC la utilización de rocas se encuentra acotada a unas pocas materias primas (gráfico 6). Al igual que en $\mathrm{CD}$, las categorías más registradas fueron sílice y basalto, dominando ampliamente las series en cada unidad de muestreo. Resultan destacables otras dos observaciones: por un lado, la presencia de obsidiana, que si bien se da en bajas frecuencias, se muestra por encima de otras rocas silíceas de uso más frecuente en la región. Por otro lado, la unidad de paisaje Meseta es la que muestra mayor variabilidad en el uso de materias primas, ya que pese a las bajas frecuencias, existe evidencia de uso de al menos $6 / 7$ de las rocas registradas en los conjuntos líticos de KK-PC.

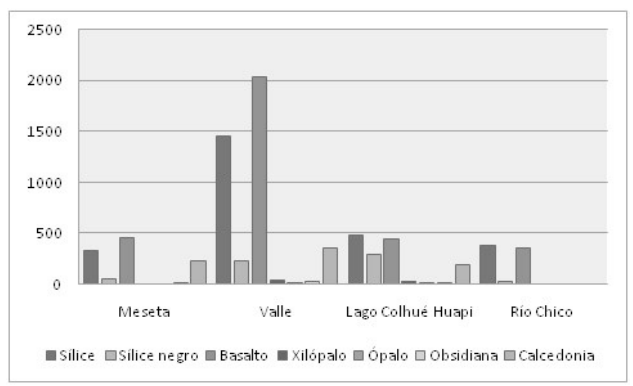

Gráfico 5. Materia prima Cerro Dragón.

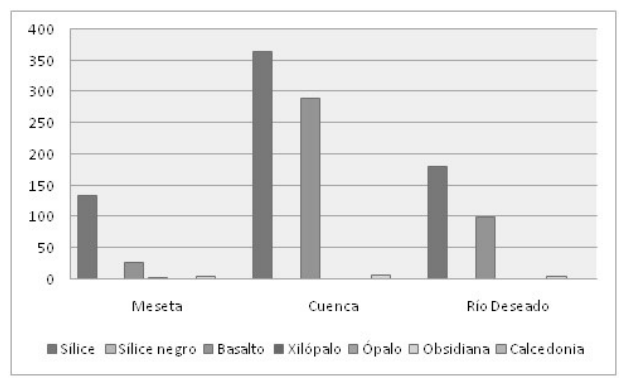

Gráfico 6. Materia prima Koluel Kaike-Piedra Clavada.

Otro de los parámetros analizados es la densidad de hallazgos artefactuales. Al comparar las densidades entre áreas, se observa que en $\mathrm{CD}$ la densidad artefactual es de 0,00060, en contraste con KK-PC que muestra una densidad de 0,00048. La evaluación de la densidad de las unidades de paisaje en cada área arroja que en ambos casos predominan densidades medias (entre 0,0002 y 0,0009 artefacto $/ \mathrm{m}^{2}$ ) y bajas (menores a 
Distribución y variabilidad del registro arqueológico de superficie en Cerro Dragón (provincia de Chubut) y Koluel Kaike-Piedra Clavada (provincia de Santa Cruz)

R. Museu Arq. Etn., 28: 70-85, 2017.

0,0002 artefacto $\left./ \mathrm{m}^{2}\right)$. Las densidades altas (mayores a 0,0009 artefacto/ $\mathrm{m}^{2}$ ) en CD están representadas por las unidades Lago Colhué Huapi y Río Chico (0,00104 y 0,00124 respectivamente), mientras que en KK-PC la única unidad de paisaje con densidad alta es Río $(0,00113)$ (Gráfico 7 y Tabla 1).

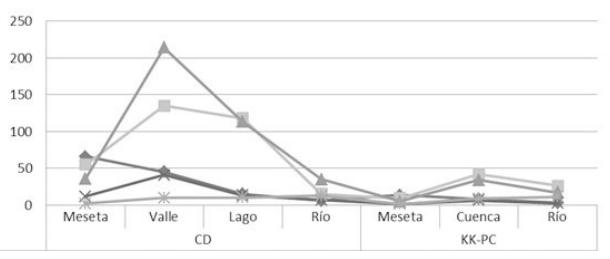

Gráfico 7. Tecnología vs densidad y $\mathrm{km}^{2} \mathrm{CD}$ y KK.

\begin{tabular}{|c|c|c|c|c|c|c|c|}
\hline & \multicolumn{4}{|c|}{$\mathrm{CD}$} & \multicolumn{3}{|c|}{ KK-PC } \\
\hline & Meseta & Valle & Lago & Río & Meseta & Cuenca & Río \\
\hline $\mathrm{m}^{2}$ relevados $(\mathrm{x} 100000)$ & 66 & 45 & 15 & 6 & 14 & 8,1 & 2,7 \\
\hline $\mathrm{AF}$ & 55 & 135 & 118 & 15 & 9 & 42 & 26 \\
\hline Núcleo & 36 & 214 & 113 & 35 & 5 & 34 & 17 \\
\hline ANF $(x 100)$ & 11,71 & 40,58 & 13,29 & 6,95 & 1,68 & 6,12 & 2,63 \\
\hline Dens $(x 1000)$ & 1,9 & 9,8 & 10,4 & 12,4 & 1,3 & 8,5 & 11,3 \\
\hline
\end{tabular}

Tabla 1. Tecnología vs densidad y $\mathrm{km}^{2} \mathrm{CD}$ y KK.

\section{Riqueza y homogeneidad}

Con el fin de comparar la distribución artefactual en ambos sectores, se obtuvieron los valores de riqueza y homogeneidad (Pielou 1977). En gráfico 8 se observa que en CD los valores de riqueza artefactual en las unidades de paisaje rondan en 0,17 y 0,22, con excepción de la unidad Lago Colhué Huapi que presenta un valor de 0,26. La homogeneidad es asimismo mayor en Lago Colhué Huapi, es decir que los elementos se encuentran repartidos de manera más equitativa entre las diferentes categorías analizadas (gráfico 8). En KK-PC, los valores de riqueza y homogeneidad muestran menor variabilidad entre las unidades paisaje, no obstante se destaca que se observa que en la unidad Río Chico los índices son mayores que en las otras dos unidades. Estos resultados dependen de la altísima frecuencia de ANF en las muestras obtenidas en cada unidad de paisaje.

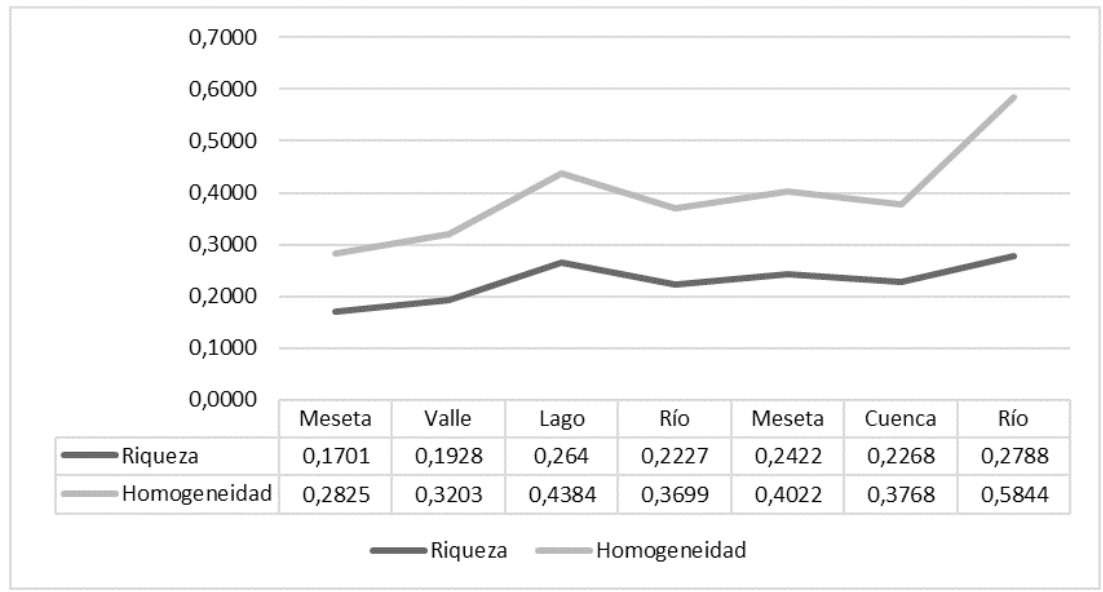

Gráfico 8. Cerro Dragón KK-PC Riqueza y homogeneidad calculada en base a clases tipológicas ANF, AF y núcleos (con lascas). 
Entonces, cabe evaluar estos parámetros excluyendo la incidencia de los ANF sobre la muestra. Para ello se calcularon los índices correspondientes utilizando la categoría núcleos y desagregando los AF en los grupos tipológicos registrados durante los trabajos de campo. De esta manera, se observa que la riqueza y homogeneidad se modifican notoriamente, produciéndose algunos cambios en las jerarquías de las unidades de paisaje analizadas. En este sentido, la unidad con mayor riqueza y homogeneidad es Lago Colhué Huapi en el caso de CD y, en el caso de KK-PC, se observa que la tendencia en la riqueza es similar a la observada en la figura anterior, aunque la homogeneidad es mucho mayor en las unidades Meseta y Cuenca. Al analizar estos parámetros según las materias primas, se observa que los conjuntos de CD poseen mayores valores de riqueza que los de KK-PC, aunque en términos de homogeneidad los valores se asemejan, con excepción de la baja homogeneidad registrada en la unidad Meseta de KK-PC (gráfico 9).

Para conocer si existe una asociación significativa entre las unidades de paisaje y las categorías analíticas utilizadas se ha llevado a cabo un test de $\mathrm{Chi}^{2}$. Los resultados obtenidos muestran que no existe ninguna asociación entre las diferentes unidades de paisaje y las categorías analizadas en este trabajo (Tabla 2)

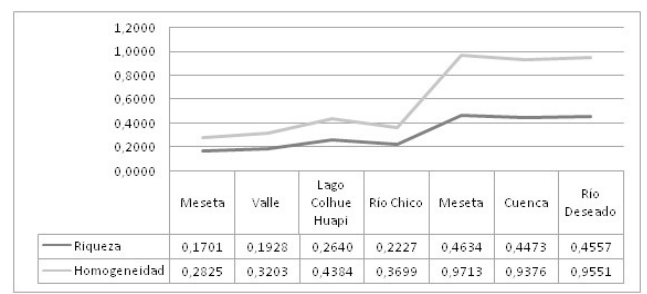

Gráfico 9. Cerro Dragón KK-PC Riqueza y homogeneidad calculada en base a grupos tipológicos (punta de proyectil, raederas, raspadores y bolas) y núcleos (sin ANFlascas).

\begin{tabular}{clccc}
\hline & & $\mathrm{x}^{2}$ & $\mathrm{gl}$ & Significación \\
\hline \multirow{4}{*}{$\mathrm{CD}$} & 19,14 & 18 &, 383 \\
& Materia prima & 23,6 & 18 &, 169 \\
\cline { 2 - 5 } KK.PC & Tecnología & 1,75 & 6 &, 941 \\
& Tipo de hallazgo & 6,87 & 10 &, 737 \\
\cline { 2 - 5 } & Materia prima & 4,57 & 8 &, 802 \\
& Tecnología & 4 &, 993 \\
\hline
\end{tabular}

Tabla 2. Prueba de $\mathrm{Chi}^{2}$ de las diferentes categorías analíticas utilizadas.

Los hallazgos de CD y KK en el contexto regional

Pese a que los objetivos son indagar sobre la distribución y características generales del registro arqueológico, creemos que es valioso poner énfasis en algunos hallazgos dado que estos aportan a la discusión de la arqueología regional. En este sentido, a continuación, enumeraremos tres tipos de artefactos. Por un lado, las puntas de proyectil son artefactos que permiten establecer diferencias entre las ocupaciones de un área particular debido a que los diferentes diseños suelen responder a determinados requerimientos de las tecnologías de armas utilizadas por diferentes grupos humanos, así como a cuestiones de estilo (Fig. 2b, c, d y e). De esta manera, conociendo la distribución de diseños en una región, las 
Distribución y variabilidad del registro arqueológico de superficie en Cerro Dragón (provincia de Chubut) y Koluel Kaike-Piedra Clavada (provincia de Santa Cruz)

R. Museu Arq. Etn., 28: 70-85, 2017.

puntas de proyectil resultan objetos sensibles a la hora de asignar cronologías relativas a las ocupaciones. En los casos analizados en CD y KK-PC, hemos discriminado al menos tres grupos morfológicos de puntas de proyectil:

(1) apendunculadas de limbo triangular;

(2) pedunculadas con aletas de tamaño mediano y (3) pedunculadas con aletas de tamaño pequeño. Las puntas apedunculadas son asimilables al estilo Magallanes III o Toldense (Bird 1938) cuya distribución temporal en Patagonia meridional corresponde al Holoceno temprano y medio (Hermo 2016). Los subgrupos de puntas pedunculadas y con aletas son semejantes a las descriptas en los complejos Magallanes IV y V y Patagoniense (Aschero 1975; Gradin, Aschero \& Aguerre, 1987), que en diversos sitios arqueológicos de Patagonia se relacionan con dataciones correspondientes al Holoceno tardío. La diferencia en tamaño podría corresponder a su utilización en distintos sistemas de armas (i.e. propulsor y dardo, arco y flecha), aunque su asignación debería basarse en parámetros métricos y morfológicos que no fueron registrados debido a las características del relevamiento. Aun así, esta diferenciación entre subgrupos permite al menos plantear que las áreas analizadas han sido ocupadas en diferentes momentos (quizás desde el Holoceno medio) aunque con mayor énfasis durante el Holoceno tardío, en consonancia con lo que muestran las investigaciones en el sur de Chubut.

Las pesas, como se expresó en el apartado de antecedentes, constituyen un tipo de artefacto que ha sido registrado en los lagos Musters y Colhué Huapi (Moreno, Pérez Ruiz \& Ramírez Rozzi 2016, Reyes \& Svoboda 2016) tanto en sitios arqueológicos como en colecciones de museos locales. Durante las prospecciones cercanas al río Chico (en el área CD) fueron hallados algunos ejemplares de pesos de red, determinados por las características de ser rodados aplanados con muescas (posiblemente de lascado simple) en los polos opuestos (Fig. 2). Cabe destacar que al igual que en los casos antecedentes, estos hallazgos se presentaron en las cercanías a fuentes de agua y distribuidos de manera aislada o en pequeñas concentraciones $(n=3)$.

Por último, destacamos el hallazgo de un instrumento formatizado con filo denticulado en el área de CD (Fig. 2f), dado lo particular del hallazgo de este tipo de artefactos en los contextos de la Patagonia meridional. En general, en las latitudes de las áreas de estudio, este grupo tipológico suele asociarse con ámbitos costeros marítimos y los estudios funcionales han mostrado que su uso se orientaba hacia el trabajo sobre hueso (Ambrústolo et al. 2011). Su registro en áreas cercanas a cursos fluviales (río Chico) amplía la distribución de estas herramientas hacia otros ambientes acuáticos.

\section{Discusión}

La información presentada en este trabajo ha sido generada a partir de datos relevados para un trabajo de impacto ambiental. Resulta interesante que a partir de este tipo de trabajos se pueda generar información de base para futuras investigaciones arqueológicas, que en áreas como las de CD y KK-PC donde la información es puntual nos permite tener un panorama más amplio acerca de la distribución y características del registro arqueológico.

En relación a lo anterior, la metodología de campo ha permitido obtener información en una escala amplia y en un tiempo relativamente corto. La utilización de transectas probabilísticas y no probabilísticas permitió abordar la distribución del registro arqueológico en ambientes en los que se a priori se detectaron rasgos del paisaje que podrían haber tenido diferentes jerarquías para el aprovechamiento humano. Los análisis realizados fueron orientados a obtener información relevante sobre cómo se distribuye el registro arqueológico en relación a esas diferencias ambientales y de esa manera obtener información acerca de las poblaciones prehistóricas que habitaron esta región.

La amplitud de los trabajos de campo permitió obtener información de una superficie relativamente alta de terreno en cada una de las 
áreas intervenidas, lo que generó un volumen de datos muy grande en base al cual podemos describir algunos patrones de distribución de los conjuntos artefactuales.

La distribución del registro arqueológico en CD y KK-PC es heterogénea. En primer lugar, la densidad artefactual es apenas superior CD que en KK-PC. En cada una de estas áreas, las unidades de paisaje con densidad alta son las más cercanas a los cuerpos de agua, Las unidades con menores densidades artefactuales son las mesetas, con frecuencias relativamente bajas de AF y Núcleos, aun cuando fueron las unidades con mayor cantidad de $\mathrm{km}^{2}$ prospectados. Por el contrario, en las unidades de paisaje ubicadas entre las mesetas y los cuerpos de agua, la densidad artefactual tiende a ser alta y en ellas se registró la mayor cantidad de AF y Núcleos.

Las características de la distribución del registro arqueológico en las unidades de paisaje nos lleva a plantear que, a esta escala de análisis, es posible identificar una determinada jerarquización del paisaje en la que los sectores más bajos, cercanos al agua y con condiciones de reparo (topográfico y/o vegetal) han sido privilegiados como sectores a el establecimiento de campamentos (i.e. bases residenciales) de manera recurrente; mientras que los sectores altos, en los que la presencia de reparo y agua son mucho menores, estuvieron destinados a actividades específicas y/o incluidas en el marco de otras tareas (i.e. abastecimiento de rocas).

De esta manera, la distribución artefactual está relacionada con la cercanía al agua, no obstante, la asociación entre el recurso crítico y el registro arqueológico no es significativa. Sin embargo, el caso del Deseado, en el que encontramos una situación inversa creo que en ese está vinculado a procesos tafonómico, porque el río y su valle de inundación está sujeto a los ciclos hídricos que durante todo el Holoceno ha mostrado diferentes grados de intensidad.

Los análisis de riqueza y homogeneidad indican que los conjuntos de KK-PC poseen valores más altos que los de $\mathrm{CD}$, información que podría definir otro punto de partida para reconocer $\mathrm{n}$ uso diferencial de ambas áreas.
Asimismo, las unidades de paisaje con valores mayores corresponden a aquellas directamente relacionadas al recurso agua, lo que creemos que muestra sintonía con la hipótesis de que en estos sectores se llevaron a cabo actividades cuyo correlato material resulta en una mayor variedad de herramientas (i.e. campamentos).

En cuanto a la forma de agrupación del registro arqueológico, la mayor presencia relativa de hallazgos aislados en las mesetas puede relacionarse con las dinámicas sedimentarias de cada unidad de paisaje, dado que las mesetas representan ambientes más estables a diferencia de las unidades de paisaje intermedias y las directamente asociadas a los cuerpos de agua, en las que los agentes tafonómicos facilitaron la visibilidad en superficie de los contextos más numerosos. No obstante, esta idea debe permanecer en calidad de hipótesis, que podrá ser puesta a prueba mediante estudios regionales específicos (Borrero 2000).

En lo que respecta al uso de materias primas líticas, hay dos variedades que muestran un uso mayoritario de las diferentes variedades de sílice y de basalto. Esta última roca ha predomina en las unidades de paisaje Meseta y Valle del área CD. Es esperable que esta preponderancia se deba a la disponibilidad natural de esta roca en los mantos basálticos que coronan a las mesetas y a su presencia en las zonas aledañas debido al transporte por remoción y por acción del agua. Los conjuntos artefactuales de CD se componen de una mayor variabilidad de rocas que los registrados en KK-PC. En esta última área, la unidad de paisaje con mayor cantidad de variedades de materias primas es la meseta. Es decir, la distribución de los diferentes tipos de materias primas registradas durante los trabajos de campo es heterogénea. Si bien discutir las causas de tal distribución excede los objetivos de este trabajo, caben destacar algunas ideas que puedan disparar futura investigaciones. Por un lado, se abre una puerta para indagar acerca de las causas de la presencia de una mayor variabilidad de rocas en $\mathrm{CD}$, que en parte se relaciona con una geología regional más variada que en KK-PC; pero que debe ser estudiada en detalle ya que 
Distribución y variabilidad del registro arqueológico de superficie en Cerro Dragón (provincia de Chubut) y Koluel Kaike-Piedra Clavada (provincia de Santa Cruz)

R. Museu Arq. Etn., 28: 70-85, 2017.

resulta llamativo que siendo un sector con disponibilidad natural de maderas petrificadas (bosque petrificado de Sarmiento), estas solo aparecen en porcentajes menores en los conjuntos líticos. Por otro lado, la presencia de obsidiana en las áreas estudiadas contribuye a las interrogantes sobre la circulación de dicha roca este sector de la Patagonia. Stern et al. (2013) señalan la presencia de variedades de obsidiana provenientes de Pampa del Asador (provincia de Santa Cruz) y de Sacanana (provincia de Chubut) en sitios del río Chico, y proponen que este curso fue parte de las rutas de conexión entre el $\mathrm{NO}$ santacruceño y el NE chubutense. La presencia recurrente de obsidiana en CD y KK-PC podría indicar el rol de estos espacios como parte de dichas rutas.

Una mirada sobre algunos tipos específicos de herramientas aporta algunos datos más para esta caracterización preliminar de las áreas estudiadas. En este sentido, la morfología de las puntas de proyectil registradas en cada una de las áreas nos ha servido para determinar la existencia de diferentes sistemas de armas, lo que a priori implicaría una cierta complejidad cronológica y cultural de relevancia para la arqueología regional. Esta variabilidad fue mayor en $\mathrm{CD}$, principalmente debido al hallazgo de puntas de proyectil triangulares apedunculadas, objetos que han sido fechados en contextos del Holoceno temprano y medio (Hermo 2016). Las pesas de red halladas en CD (río Chico) amplían la distribución registrada para este tipo de artefactos, complementando las muestras estudiadas por otros investigadores en el área de los lagos Musters y Colhué Huapi (Svoboda \& Reyes 2014; Moreno, Pérez Ruiz \& Ramírez Rozzi 2016). Otro caso que quisimos resaltar fue el hallazgo de un raspador denticulado en $C D$, debido a la singularidad del hallazgo en las prospecciones realizadas ya que los reportes acerca de este grupo tipológico hacia el interior de Patagonia no suelen ser frecuente. Lejos de intentar profundizar en encontrar respuestas para el significado de la presencia de un raspador denticulado en el área señalada, lo que nos interesa es señalar dicho objeto como parte de la complejidad del registro arqueológico de las áreas presentadas.
A modo de conclusión, remarcamos algunos elementos que evaluamos como aporte de este trabajo a la arqueología de Patagonia. Se ha mostrado que a partir de trabajos de impacto arqueológico es posible construir información de base que pueda ser de provecho para otros arqueólogos. En los casos presentados se contó con datos de poca profundidad, pero representativos de una superficie muy importante. La difusión de este tipo de información en los ámbitos de difusión científica permite incorporar datos que de otra manera circularían en los ámbitos en los que se mueven los informes técnicos, con una difusión claramente menor.

De esta manera, se brindó una caracterización inicial de la distribución del registro arqueológico en las áreas estudiadas, que comienza a llenar un vacío de información. Estos trabajos representan un punto de partida para investigar las maneras es que el SE chubutense y el NE santacruceño fueron poblados en el pasado y las hipótesis propuestas deberán ser estudiadas en detalle dado su carácter preliminar. En este sentido, CD y KK-PC son áreas que muestran diferencias ambientales y en la distribución del registro arqueológico en las unidades de paisajes. Se propuso que una de las causas sería la jerarquización del paisaje en relación a la ubicación de fuentes de agua y de otros recursos (materias primas líticas, vegetales, reparo). Tal jerarquización se materializa en forma de áreas en las que predominan conjuntos relativamente numerosos y con una variabilidad de herramientas relativamente importante, lo que indica posibles áreas de campamento; frente a otros sectores en los que las características del registro denotan actividades acotadas. La presencia de algunos tipos de herramientas y su variabilidad indica que el uso humano del área puede ser previa al Holoceno tardío y que existe un registro lítico que muestra conexiones con otros sectores (obsidiana, raspador denticulado, pesas) cuyo estudio en detalle aportará al conocimiento de la dinámica de circulación de objetos a escala regional. Esta caracterización inicial de las áreas CD y KK-PC debe ser considerado como un primer intento 
de generación de información de base para futuras investigaciones y para comparación con otras áreas.

\section{Agradecimientos}

Deseamos expresar nuestro agradecimiento a la ayuda durante las tareas de campo, a los integrantes de la División Arqueología - Museo de Ciencias Naturales de La Plata, Dres. Enrique Terranova, Bruno Mosquera y Rocío Blanco. A la Ing. Elena Vicente y la Ing. Magdalena Quirno-Costa (Medio Ambiente Panamerican Energy), al lic. Julio Cotti Alegre, lic. Marina San Martin y Sr. Sebastián Fonzalida (Hidroar S.A.). Agradecemos la desinteresada ayuda prestada por el lic. Juan Diego Gobbo (División Arqueología - Museo de La Plata).

DEL PAPA, M.C.; HERMO, D. Distribution and variability of the surface archaeological record in Cerro Dragón (Chubut province) and Koluel Kaike-Piedra Clavada (Santa Cruz province). R. Museu Arq. Etn., 28: 70-85, 2017.

\begin{abstract}
The studies that are framed within the denominated contract archeology, specifically those related to studies of environmental impact, have reviewed in recent years an important source of information on the characteristics of the archaeological heritage of different regions of the Argentine Republic. In this context, the results presented in this work belong to the Baseline Study corresponding to two areas of oil exploitation. These areas are located in the San Jorge Hydrocarbon Basin (Cerro Dragón, Chubut province, and Koluel Kaike-Piedra Clavada, Santa Cruz province). The information obtained, as a product of the surface survey allowed to perform a set of analyzes that allowed the characterization of the archaeological record of an important area over which there is practically no antecedents. We analyzed the distribution of different types of findings, the raw materials used and aspects of the technologies represented in the sample. We calculated the richness and homogeneity and the possible association between different landscape units and the different analytical categories used. The obtained results show the existence of a distribution of the archaeological record in surface that is expressed of heterogenous form between the different units of the landscape analyzed, being the greater densities in those areas near critical resources such as water sources.
\end{abstract}

Keywords: Contract archeology; Patagonia; San Jorge Gulf; Cerro Dragón; Koluel Kaike-Piedra Clavada.

\title{
Referências bibliográficas
}

Ambrústolo, P. et al. 2011. Instrumentos líticos con filos denticulados en la Costa Norte de Santa Cruz: un análisis tecno-funcional. Cazadoresrecolectores del Cono Sur 4: 79-94.

Aschero, C. 1975. Secuencia arqueológica del Alero de las Manos Pintadas: Las Pulgas, departamento
Río Senguerr, Chubut. Relaciones de la Sociedad Argentina de Antropología 9: 187-209.

Aschero, C.; Manzi L.; Gómez A.G. 1994. Producción lítica y uso del espacio en el nivel 2b4 de Quebrada Seca 3. Relaciones de la Sociedad Argentina de Antropología 19: 191-214. 
Distribución y variabilidad del registro arqueológico de superficie en Cerro Dragón (provincia de Chubut) y Koluel Kaike-Piedra Clavada (provincia de Santa Cruz)

R. Museu Arq. Etn., 28: 70-85, 2017.

Belardi, J.B. 2005. Paisajes arqueológicos: un estudio comparativo de diferentes ambientes patagónicos. Archaeopress, Oxford.

Bird, J. 1938. Antiquity and migrations of the early inhabitants of Patagonia. Geographical Review 28(2): 250-275.

Borrero, L.A. 2000. Ten Years After: esquema para una tafonomía regional de la Patagonia meridional y Norte de Tierra del Fuego. In: Espinosa, S. Desde el País de los Gigantes: perspectivas arqueológicas en Patagonia. Universidad Nacional de la Patagonia Austral, Río Gallegos, tomo I, 183-193.

Borrero, L.A.; Lanata, J.L.; Ventura, B.N. (1992) Distribuciones de hallazgos aislados en Piedra del Águila. In: Borrero, L.A.; J.L. Lanata (Orgs.). Análisis espacial en la Arqueología Patagónica. Ayllu, Buenos Aires, 9-20.

Bozzuto D.L. 2013. Análisis de material lítico superficial en el noreste del lago PueyrredónCochrane (provincia de Santa Cruz, Argentina). Revista Española de Antropología Americana 43(1): 73-88.

Cardich, A.R.; Cardich, L.A.; Hajduk, A. 1973. Secuencia arqueológica y cronológica radiocarbónica de la Cueva 3 de Los Toldos (Santa Cruz, Argentina). Relaciones de la Sociedad de Antropología 7: 85-123.

Dunnell, R.C.; Dancey, W.S. 1983. The siteless survey: a regional scale data collection strategy. Advances in Archaeological Method and Theory 6: 267-287.

Ebert, J.I. 1992. Distributional archaeology. University of New Mexico, Albuquerque.

Foley, R. 1981. A model of regional archaeological structure. Proccedings of the Prehistoric Society 47: $1-17$.

Gallardo, F.A.; Cornejo L.E. 1986. El diseño de la prospección arqueológica: un caso de estudio. Chungará 16-17: 409-420.
Gradin, C.J. 1973. La piedra pintada de Mamuel Choique (Provincia de Río Negro). Relaciones de la Sociedad Argentina de Antropología 7: 145-157.

Gradin, C.J.; Aguerre A.M. 1994. Excavación del enterratorio de Puesto el Rodeo. In: Gradin, C.J.; Aguerre, A.M. (Orgs.). Contribución a la arqueología del río Pinturas. Ayllu, Buenos Aires, 259-272.

Gradin, C.J.; Aschero, C.A.; Aguerre, A.M. 1987. Primeros niveles culturales en el Área Río Pinturas (Provincia de Santa Cruz, Argentina). Estudios Atacameños 8: 118-141.

Guráieb, A.G. 1999. Análisis de la diversidad en los conjuntos instrumentales liticos de Cerro de los Indios 1 (Lago Posadas, Santa Cruz). Relaciones de la Sociedad Argentina de Antropología 24: 293-306.

Guraieb, S.G. et al. 2009. Nuevos estudios del esqueleto del sitio Cerro Yanquenao (Colhue Huapi, Chubut): veintiocho años después. Magallania 37(2): 165-175.

Hermo, D. 2016. Variabilidad morfológica y cronología en puntas triangulares apedunculadas del Macizo del Deseado (provincia de Santa Cruz). In: Actas del XIX Congreso Nacional de Arqueología Argentina, 2016, San Miguel de Tucumán, 3073-3076.

Herrera, V.M.; Cuenca, E.C.; Pérez, S.C. 2009. Métodos de prospección arqueológica intensiva en el marco de un proyecto regional: el caso de la comarca de La Serena (Badajoz). Trabajos de Prehistoria 66(1): 7-26.

Judge, W.J.; Ebert J.I.; Hitchcock R.K. 1975. Sampling in regional archaeological survey. In: Mueller, J.W. (Org.). Sampling in archaeology. University of Arizona, Tucson, 82-123.

Menghin, O. 1952. Fundamentos cronológicos de la prehistoria de Patagonia. Runa 5: 23-43.

Miotti, L. 1996. Piedra Museo (Santa Cruz), nuevos datos para la ocupación pleistocénica en 
Patagonia. In: Otero, J.G. (Org.). Arqueología: solo Patagonia. Centro Nacional Patagónico, Puerto Madryn, 27-38.

Miotti, L. et al. 2014. Fechados radiocarbónicos y delimitación temporal de los conjuntos arqueológicos de Cueva Maripe, Santa Cruz (Argentina). Relaciones de la Sociedad Argentina de Antropología 39(2): 509-537.

Miotti, L.; Salemme M.C. 2003. When Patagonia was colonized: people, mobility at high latitudes during Pleistocene/Holocene transition. Quaternary International 109-110: 95-111.

Moreno, E.; Pérez Ruiz H. 2010. Evidencias de utilización prehispánica de recursos fluviales en la cuenca del lago Musters (Chubut, Argentina). In: Actas del XVII Congreso Nacional de Arqueología Argentina. Facultad de Filosofia y Letras, Universidad Nacional de Cuyo, Mendoza, 2010, 345-350.

Moreno E.; Pérez Ruiz, H.; Ramírez Rozzi F. 2016. Esquema cronológico y evolución del paisaje en el bajo Sarmiento (Chubut). In: Mena, F. (Org.). Arqueología de la Patagonia: de mar a mar. Ciep; Nire Negro, Santiago de Chile, 477-486.

Outes, F.F. 1905. La Edad de Piedra en Patagonia: estudio de arqueología comparada. Anales del Museo Nacional de Buenos Aires 5: 204-375.

Pérez de Micou, C.P. 2009 Tejidos para la vida y la muerte: Pérez de rendas textiles en el Alero Mazquiarán. In: Pérez de Micou, C.P.; Mandri, M.T.; Burry, L.S. (Orgs.). Imágenes desde un alero: investigaciones multidisciplinares en Río Mayo, Chubut, Patagonia argentina. Fundación de Historia Natural Félix de Azara, Buenos Aires, 81-92.

Pielou, C. 1977. Mathematical ecology. John Wiley, New York.

Plog, S.; Plog, F.; Wait, W. 1978. Decision making in modern surveys. Advances in Archaeological Method and Theory 1: 383-421.
Ratto, N.; Carniglia, D. 2013. Propiedades del registro y tafonomía de conjuntos líticos: el caso del norte de la Provincia de Santa Cruz (Argentina). In: Zangrando, A.F. et al. (Orgs.). Tendencias teóricometodológicas y casos de estudio en la arqueología de la Patagonia. Museo de Historia Natural de San Rafael, San Rafael, 485-494

Reyes, M.; Svoboda, A. 2016. Un acercamiento a las artes de pesca a partir del análisis de los pesos líticos en el área de los lagos Musters y Colhúe Huapi (Provincia de Chubut). In: Mena, F. (Org.). Arqueología de la Patagonia: de mar a mar. Ciep; Nire Negro, Santiago de Chile, 496-509.

Scheinsohn, V. 2001. 2001: odisea del espacio: paisajes y distribuciones artefactuales en arqueología: resultados y propuestas. Relaciones de la Sociedad Argentina de Antropología 26: 285-302.

Shannon, C.E.; Weaver, W. 1949. The mathematical theory of communication. University Illinois, Urbana.

Stern, C.R.; Castro A.; Pérez de Micou, C.; Méndez, C.; Mena, F. 2013. Circulación de obsidianas en Patagonia Central-Sur entre 44 y $46^{\circ} \mathrm{S}$. In: Zangrando, A.F.; Barberena, R.; Gil, A.; Neme, G.; Giardina, M.; Luna, L.; Otaola, C.; Paulides, S.; Salgán, L.; Tivoli; A. Tendencias teórico-metodológicas y casos de estudio en la arqueología de la Patagonia. Museo de Historia Natural de San Rafael; INAPL, San Rafael, 243-250.

Vignati, M. 1950. Estudios antropológicos en la zona militar de Comodoro Rivadavia. Universidad de La Plata, La Plata.

Zar, J.H. 1974. Biostatistical analysis. Prentice Hall, Englewood Cliffs.

Zubimendi, M.; Moreno, J.E. 2014. La presencia de artefactos arqueomalocológicos en la localidad arqueológica Delta del arroyo Vulcana (lago Musters, provincia de Chubut). Intersecciones en antropología 15(1): 71-87. 